Erratum

\title{
Erratum: Polge, C., et al. UBE2E1 Is Preferentially Expressed in the Cytoplasm of Slow-Twitch Fibers and Protects Skeletal Muscles from Exacerbated Atrophy upon Dexamethasone Treatment. Cells 2018, 7,214
}

Cécile Polge ${ }^{1}$, Julien Aniort ${ }^{1}$, Andrea Armani ${ }^{2}$, Agnès Claustre ${ }^{1}$, Cécile Coudy-Gandilhon ${ }^{1}$, Clara Tournebize ${ }^{1}$, Christiane Deval ${ }^{1}$, Lydie Combaret ${ }^{1}$, Daniel Béchet ${ }^{1}{ }^{\circledR}$, Marco Sandri ${ }^{2}$, Didier Attaix ${ }^{1}$ and Daniel Taillandier ${ }^{1, *}$

1 INRA, UMR 1019, Human Nutrition Unit (UNH), 63122 St Genès Champanelle, France; cecile.polge@inra.fr (C.P.); j.aniort@orange.fr (J.A.); agnes.claustre@inra.fr (A.C.); cecile.coudy-gandilhon@inra.fr (C.C.-G.); clara.tournebize@gmail.com (C.T.); christiane.deval@inra.fr (C.D.); lydie.combaret@inra.fr (L.C.); daniel.bechet@inra.fr (D.B.); didier.attaix@inra.fr (D.A.)

2 Venetian Institute of Molecular Medicine, 35100 Padova, Italy; andre.arma88@gmail.com (A.A.); marco.sandri@unipd.it (M.S.)

* Correspondence: daniel.taillandier@inra.fr; Tel.: +33-473-62-48-44

Received: 29 November 2018; Accepted: 30 November 2018; Published: 4 December 2018

check for updates

Change in author names (order).

The authors wish to make the following corrections to this paper [1]:

Cécile Polge ${ }^{1}$, Julien Aniort ${ }^{1}$, Andrea Armani ${ }^{2}$, Agnès Claustre ${ }^{1}$, Cécile Coudy-Gandilhon ${ }^{1}$, Clara Tournebize ${ }^{1}$, Christiane Deval ${ }^{1}$, Lydie Combaret ${ }^{1}$, Daniel Béchet ${ }^{1}$, Marco Sandri ${ }^{2}$, Didier Attaix ${ }^{1}$ and Daniel Taillandier ${ }^{1, *}$

1 INRA, UMR 1019, Human Nutrition Unit (UNH), 63122 St Genès Champanelle, France; cecile.polge@inra.fr (C.P.); j.aniort@orange.fr (J.A.); agnes.claustre@inra.fr (A.C.); cecile.coudy-gandilhon@inra.fr (C.C.-G.); clara.tournebize@gmail.com (C.T.); christiane.deval@inra.fr (C.D.); lydie.combaret@inra.fr (L.C.); daniel.bechet@inra.fr (D.B.); didier.attaix@inra.fr (D.A.)

2 Venetian Institute of Molecular Medicine, 35100 Padova, Italy; andre.arma88@gmail.com (A.A.); marco.sandri@unipd.it (M.S.)

The authors would like to apologize for any inconvenience caused to the readers by these changes. The changes do not affect the scientific results.

\section{References}

1. Polge, C.; Aniort, J.; Armani, A.; Claustre, A.; Coudy-Gandilhon, C.; Tournebize, C.; Deval, C.; Combaret, L.; Béchet, D.; Sandri, M.; et al. UBE2E1 Is Preferentially Expressed in the Cytoplasm of Slow-Twitch Fibers and Protects Skeletal Muscles from Exacerbated Atrophy upon Dexamethasone Treatment. Cells 2018, 7, 214.

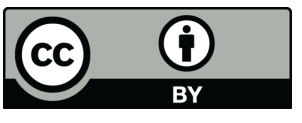

(C) 2018 by the authors. Licensee MDPI, Basel, Switzerland. This article is an open access article distributed under the terms and conditions of the Creative Commons Attribution (CC BY) license (http:/ / creativecommons.org/licenses/by/4.0/). 